\title{
Enhancement of transfection efficiency with NLS and SPB-NLS
}

\author{
JIAN LIN, TAO QIN, LI QI ZHU, QIANG ZHANG, QING HUA YU and QIAN YANG \\ Key Laboratory of Animal Physiology and Biochemistry, Ministry of Agriculture, Nanjing Agricultural University, \\ Nanjing, Jiangsu 210095, P.R. China
}

Received July 18, 2013; Accepted March 3, 2014

DOI: $10.3892 / \mathrm{mmr} .2014 .2121$

\begin{abstract}
Low positive cell screening efficiency severely hinders the development of transgenic animals. The major rate-limiting step of positive cell screening is DNA entering the nucleus, particularly for large DNA molecules. To enhance the transport of large DNA molecules into the nucleus, particularly for the production of transgenic animals, nuclear localization sequence (NLS) peptides and the peptide derivative succinimid yl-[4-(psoralen-8-yloxy)]-butyrate (SPB)-NLS were synthesized to mediate transfection in vitro. To investigate the function of NLS and SPB-NLS in vitro, the expression levels of growth hormone $(\mathrm{GH})$ mRNA and green fluorescent protein (GFP) protein were analyzed following transfection mediated by NLS and SPB-NLS. The results demonstrated that the expression of GH mRNA was significantly higher in the NLS (increased by $69 \%$ ) and SPB-NLS (330\%) groups than that in the liposome/ pGN group. Similarly, GFP expression was found to be higher in the SPB-NLS group than that in the liposome group, while the expression in the NLS group was lower than that in the liposome group. Further analysis demonstrated that SPB-NLS enhanced the expression of insulin-like growth factor 1 in hard-to-transfect goat mammary epithelia cells. The results of the microscopy analysis revealed that transfected DNA entered the nucleus via the nuclear pores, facilitated by NLS. Analysis of the cell cycle demonstrated that the cytotoxic effects of NLS and SPB-NLS were low. In conclusion, the results of the present study demonstrate that SPB-NLS acts as a transfection-enhancing agent and may be used both to enhance nuclear delivery and for the development of genetically modified animals.
\end{abstract}

\section{Introduction}

The development of transgenic animals is a promising technology in numerous fields; however, low production efficiency is a significant limiting factor (1). Rapid and efficient screening

Correspondence to: Professor Qian Yang, Key Laboratory of Animal Physiology and Biochemistry, Ministry of Agriculture, Nanjing Agricultural University, Weigang 1, Nanjing, Jiangsu 210095, P.R. China

E-mail: zxbyq@njau.edu.cn

Key words: low screen efficiency, large molecular DNA delivery, transfection enhancement, nuclear location, GMEC expression of positive clones of genetically modified cells may accelerate the development of transgenic animals. To produce transgenic animals, exogenous DNA must first enter the cell and then the nucleus, prior to integrating into the chromosomal DNA. To achieve safe and efficient delivery of plasmid DNA using a non-viral gene delivery system, the most important cell barrier to overcome is the nuclear membrane $(2,3)$. The functional diameter of the nuclear pore is $\sim 10 \mathrm{~nm}$ (4). The efficiency of nuclear import is considered to be an important kinetic block that influences transgenic expression efficiency (5). Due to the low efficiency rate of the integration of plasmid DNA into chromosomal DNA, focus has been placed on improving transfection efficiency by enhancing the efficiency of transport through the nuclear pore (6).

In order to enhance transfection efficiency, one strategy is to attach a nuclear localization sequence (NLS) peptide to the plasmid DNA to facilitate nuclear localization and therefore improve gene expression efficiency in target tissues (7). Subramanian et al (8) demonstrated that, by conjugating an NLS peptide to DNA, there was an increase in reporter gene expression (8). However, other previous studies that have chemically conjugated NLS to DNA to guide transport into the nucleus have found that this does not significantly improve transfection efficiency, and in certain cases the efficiency was even observed to decrease due to chemical modification of the DNA, leading to reduced transcription efficiency in the nucleus $(9,10)$. In an attempt to solve this problem, bi- (11) and tri-functional (12) synthetic peptides have been designed to improve gene transfer efficiency. Succinimidyl-[4-(psoralen-8-yloxy)]-butyrate (SPB), a DNA intercalating reagent, has been used to non-covalently modify NLS moieties to enhance transgenic expression efficiency.

The optimal conditions for SPB-NLS peptides to enhance gene transfer efficiency have yet to be elucidated. Only upon entering the nucleus is a plasmid able to be expressed. The aim of the present study was to confirm the function of the NLS peptide and the peptide derivative SPB-NLS in enhancing the transfection efficiency of large DNA molecules (plasmids pIN and pGN). The elucidation of this function may reduce the problems associated with low screen efficiency in the development of transgenic animals.

\section{Materials and methods}

Materials and reagents. SPB was obtained from Pierce Biotechnology Inc. (Rockford, IL, USA). Dulbecco's 
Modified Eagle's Medium (DMEM), DMEM/F-12, fetal bovine serum (FBS) and liposome (Lipofectamine 2000) were obtained from Invitrogen Life Technologies (Carlsbad, CA, USA). Radioimmunoprecipitation assay (RIPA) buffer [0.1\% (w/v) SDS, $1 \%(\mathrm{v} / \mathrm{v})$ Triton X-100, $1 \%(\mathrm{w} / \mathrm{v})$ sodium deoxycholate in Tris-buffered saline (TBS; $25 \mathrm{mM}$ Tris/HCl, pH 7.5 and $150 \mathrm{mM} \mathrm{NaCl}$ )], and phosphatase and protease inhibitors [1 mM phenylmethylsulfonyl fluoride (PMSF), $1 \mathrm{mM} \mathrm{Na}_{3} \mathrm{VaO}_{4}$ and $\left.25 \mathrm{mM} \mathrm{NaF}\right]$ were obtained from the Beyotime Institute of Biotechnology (Haimen, China). Horseradish peroxidase (HRP)-conjugated goat anti-mouse immunoglobulin $\mathrm{G}$ ( $\mathrm{IgG}$ ) and rabbit anti-mouse $\mathrm{IgG}$ were obtained from Bioworld Technology, Inc. (St. Louis Park, MN, USA). Antibodies specific to green fluorescent protein (GFP) and monoclonal bovine $\beta$-actin were obtained from Cell Signaling Technology, Inc. (Danvers, MA, USA). Mouse monoclonal antibody against insulin-like growth factor 1 (IGF-1) was obtained from Abcam (Cambridge, MA, USA). Phalloidin-fluorescein isothiocyanate, DAPI and RNaseA were obtained from Sigma (St. Louis, MO, USA). All other chemicals and reagents used were of analytical grade. The present study was approved by the ethics committee of Nanjing Agriculture University (Nanjing, Jiangsu, China) and the Jiangsu Provincial Academy of Agricultural Sciences. The license number was SCXK (Su) 2002-0029.

Plasmid DNA. Plasmid DNA (pAC-GFP-N1) was provided by the College of Life Sciences, Nanjing Agricultural University (Nanjing, China). Plasmid DNA pGN and pIN (13) were constructed in our lab (Key Laboratory of Animal Physiology and Biochemistry, Nanjing, China). A map of the vectors and a basic description are shown in Fig. 1. Plasmid DNA was amplified by Escherichia coli DH5a and purified using E.Z.N.A ${ }^{\circledR}$ Endo-Free Plasmid Mini Kit I (Omega Bio-tek $^{\circledR}$, Norcross, GA, USA). The purity of the plasmids was determined using electrophoresis on a $1.0 \%$ agarose gel, and the concentration of DNA was determined by measuring the ultraviolet absorbance at 260 and $280 \mathrm{~nm}$.

Cell line. The human breast cancer cell line Bcap-37 (estrogen receptor negative, p53 mutated) was obtained from Shanghai Cell Collection, Chinese Academy of Sciences (Shanghai, China). The Bcap-37 cell line is difficult to transfect, and was therefore appropriate for the study of gene transfer efficiency. Bcap-37 cells were grown in DMEM supplemented with $10 \%$ FBS at $37^{\circ} \mathrm{C}$ in a humidified atmosphere of $5 \% \mathrm{CO}_{2}$.

Mammary epithelial cell isolation and culture. Mammary gland tissues were obtained from Saanen dairy goats (Capra hircus). Cells were grown in DMEM/F-12 with 10\% FBS in the presence of penicillin $(100 \mathrm{U} / \mathrm{ml})$ and streptomycin $(100 \mathrm{mg} / \mathrm{ml})$ under standard culture conditions $(5 \%$ $\left.\mathrm{CO}_{2}, 37^{\circ} \mathrm{C}\right)$. Mammary epithelial cell culture was performed as described previously (14).

Preparation of complex formation and evaluation of optimal conditions.

$S P B$ conjugation with $N L S$. To take advantage of cellular import machinery NLS peptide and peptide derivative SPB-NLS were synthesized by Sangon Biotech Shanghai Co., Ltd. (Shanghai,

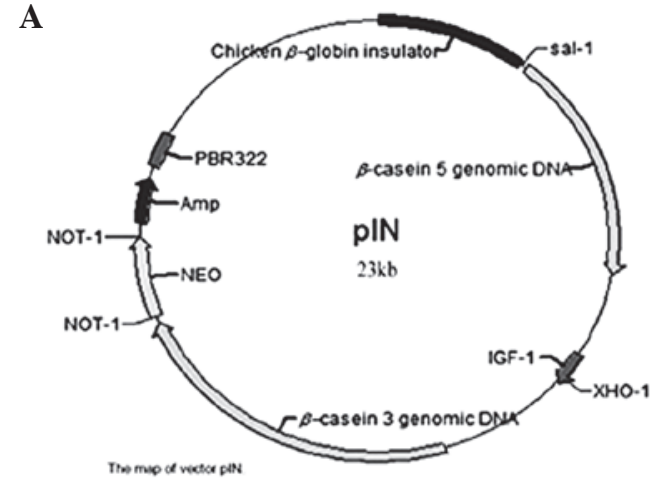

B

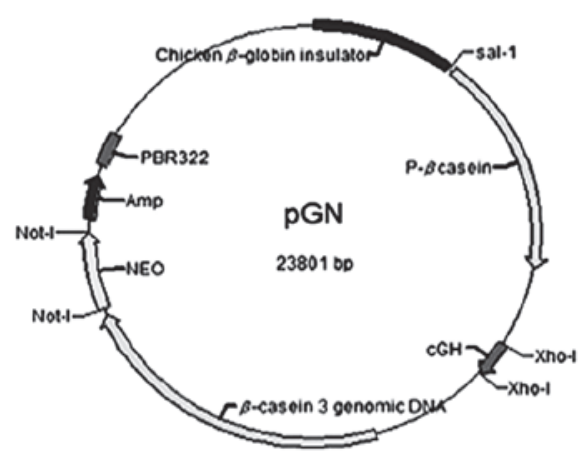

C

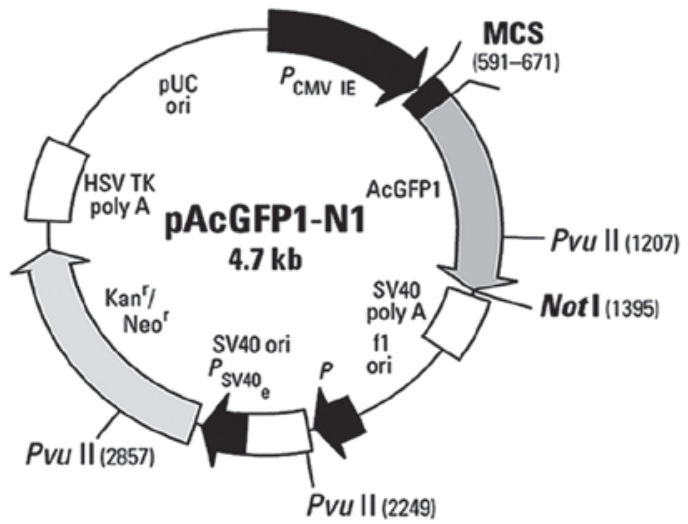

Figure 1. Vector map of plasmids. (A) Plasmid pIN was constructed by our laboratory (Key Laboratory of Animal Physiology and Biochemistry, Nanjing, China) for specific expression of IGF-1 in mammary glands to increase milk production. Plasmid pIN was constructed using the bone plasmid $\mathrm{pBC1}$, a 21.6-kb vector designed to facilitate the expression of recombinant protein in milk of transgenic animals. Plasmid pIN was a $23.6-\mathrm{kb}$ vector that was too large to be transfected. (B) Plasmid pGN was constructed by our laboratory for specific expression of GH in mammary glands to increase milk production. Plasmid pIN was also constructed using the bone plasmid $\mathrm{pBC1}$. Plasmid pIN was a $23.8-\mathrm{kb}$ vector that was too large to be transfected. (C) pAcGFP1-N1 encoded GFP from Aequorea coerulescens (excitation maximum $=475 \mathrm{~nm}$; emission maximum $=505 \mathrm{~nm}$ ). The coding sequence of the AcGFP1 gene contained silent base changes, which corresponded to human condon-usage preferences. IGF-1, insulin-like growth factor 1; GH, growth hormone; GFP, green fluorescent protein; MCS, multiple cloning site.

China) using the following sequences: CGGPKKKRKVP (classical NLS) and SPB-PKKKRKV. Amine-reactive SPB was used during NLS peptide synthesis to obtain SPB-terminated NLS conjugates. Purification was performed using reverse-phase chromatography. High, pure grades of conjugates were selected with $\geq 98 \%$ high-performance liquid 
Table I. qPCR primers used to detect plasmid pGN.

\begin{tabular}{llll}
\hline Gene & Sense primer & Antisense primer & Product length (bp) \\
\hline $\mathrm{GH}$ & gagaagctgaaggacctgga & tacgtctccgtcttgtgcag & 194 \\
Bcap-37 $\beta$-actin & gatcattgctcctcctgagc & tgtggacttgggagaggact & 385 \\
\hline
\end{tabular}

qPCR, quantitative polymerase chain reaction; $\mathrm{GH}$, growth hormone.

chromatography purity in accordance with the manufacturer's own quality control.

Preparation of NLS/DNA and SPB-NLS/DNA complexes. A total of $2 \mathrm{mg}$ NLS $(1.67 \mu \mathrm{mol}$; molecular weight, 1,195.53) was first dissolved in $167 \mu \mathrm{l}$ dimethyl sulfoxide (DMSO) solution, and 1,500 $\mu 120 \mathrm{mM}$ 2-[4-(2-hydroxyethyl)piperazin-1-yl]ethanesulfonic acid (HEPES) solution was then added and agitated for $30 \mathrm{~min}$; the final concentration of NLS solution was $1 \mathrm{pM}$. In addition, $2 \mathrm{mg}$ SPB-NLS $(1.73 \mu \mathrm{mol}$; molecular weight, 1,153.42) was dissolved in $173 \mu \mathrm{l}$ DMSO solution, and $1500 \mu 120 \mathrm{mM}$ HEPES solution was then added and agitated for $30 \mathrm{~min}$; the final concentration of SPB-NLS solution was $1 \mathrm{pM}$. NLS/DNA and SPB-NLS/DNA complexes were prepared at various molar ratios by adding different amounts of NLS or SPB-NLS solution into fixed DNA (plasmid pGN) solution $(1 \mu \mathrm{g})$. The molar ratio of the NLS/DNA and SPB-NLS/DNA complexes ranged between $5 \times 10^{2}$ and $5 \times 10^{4}$. Mixtures were gently agitated at room temperature for $30 \mathrm{~min}$ to initiate formation.

Gel retardation assay. Different amounts of NLS and SPB-NLS solutions (molar ratio, between $5 \times 10^{2}$ and $5 \times 10^{4}$ ) were combined with DNA solution ( $1 \mu \mathrm{g}$ plasmid pGN). The function of condensing plasmid DNA by NLS or SPB-NLS was measured using electrophoresis. Gels were prepared with $1 \%$ agarose in Tris-acetate-EDTA buffer with ethidium bromide. Electrophoresis was performed for $30 \mathrm{~min}$ at $150 \mathrm{~V}$. The volume of samples loaded was $10 \mu 1$.

Analysis of the transfection efficiency of NLS and SBP-NLS Quantitative polymerase chain reaction ( $q P C R$ ) analysis. Bcap-37 cells were incubated at $37^{\circ} \mathrm{C}$ for $48 \mathrm{~h}$ prior to transfection. Transfection was performed using plasmid pGN mediated by NLS and SPB-NLS to evaluate the transfection efficiency. Primers used are listed in Table I. NLS/DNA and SPB-NLS/DNA complexes were prepared as mentioned above. Liposome solution was added to complexes and incubated for a further $20 \mathrm{~min}$ at room temperature prior to transfection. Medium was changed to complete medium $6 \mathrm{~h}$ after transfection. Growth hormone $(\mathrm{GH})$ gene expression level was analyzed using qPCR at $48 \mathrm{~h}$. All transfection experiments were performed in triplicate.

Fluorescence microscopy and flow cytometric analysis. The plasmid pAC-GFP-N1 (expressing GFP as a reporter gene) was transfected into Bcap-37 cells with the aid of NLS or SPB-NLS to evaluate their bioactivity. Approximately 10,000 cells/population were plated on 12-well dishes (Costar ${ }^{\circledR}$; Corning, Inc., Corning, NY, USA) and incubated at $37^{\circ} \mathrm{C}$ for $24 \mathrm{~h}$ prior to transfection. Transfection was performed in accordance with the aforementioned method.
Following transfection, cells were incubated for $48 \mathrm{~h}$ at $37^{\circ} \mathrm{C}$ and then washed twice with phosphate-buffered saline (PBS). Images were captured by standard fluorescence microscopy (Olympus, Tokyo, Japan).

Preliminary results from the fluorescence microscopy revealed high levels of GFP expression in the SPB-NLS-mediated group. Therefore, the relative increase in expression was calculated using flow cytometry, since this method allows the rapid analysis a large number of cells. Transfection of Bcap-37 cells was performed in accordance with the aforementioned method using the plasmid pAC-GFP-N1. Cells were harvested $48 \mathrm{~h}$ after transfection, washed three times with PBS and then suspended in $500 \mu \mathrm{l}$ cold PBS and examined using a BD FACSCalibur ${ }^{\mathrm{TM}}$ flow cytometer (BD Biosciences, Franklin Lakes, NJ, USA) equipped with an argon laser (488 $\mathrm{nm})$.

Western blot analysis. Bcap-37 cells were transfected with plasmid pAC-GFP-N1 to further investigate the function of SPB-NLS. Transfection was performed in accordance with the aforementioned method. Cells were harvested 24 and $48 \mathrm{~h}$ after transfection and lysed using RIPA buffer containing phosphatase and protease inhibitors (1 mM PMSF) on ice for $30 \mathrm{~min}$. Cell lysates were then centrifuged at $14,000 \mathrm{x} \mathrm{g}$ for $10 \mathrm{~min}$ at $4^{\circ} \mathrm{C}$ and the concentration of the proteins in the supernatant was determined using a bicinchoninic acid protein assay kit (Pierce Biotechnology Inc.). The proteins were separated using 4-15\% SDS-PAGE and transferred onto nitrocellulose membranes. The membranes were probed using an anti-GFP primary antibody $(1: 2,500)$, followed by a goat anti-mouse IgG-HRP secondary antibody. Protein expression was detected using an enhanced chemiluminescence detection system (Amersham Pharmacia Biotech, Inc., Amersham, UK). $\beta$-actin was used as a loading control. All bands from the western blotting were analyzed using Quantity One software (Bio-Rad, Hercules, CA, USA) to verify their relative expression.

Confocal microscopy to investigate plasmid DNA localization. To investigate whether NLS and SPB-NLS enhanced plasmid transfection to the nucleus, plasmid pGN was labeled using a $\mathrm{Cy}^{\mathrm{TM}} 3$ labeling kit (Mirus Bio LLC, Madison, WI, USA). Transfection was performed in accordance with the aforementioned method. Bcap-37 cells were divided into five groups [blank, liposome, NLS, SPB-NLS and wheat-germ agglutinin (WGA)/SPB-NLS]. WGA was used to specifically block the nuclear pores. Cells were washed three times with PBS $2 \mathrm{~h}$ after transfection and then fixed using $4 \%$ paraformaldehyde for $30 \mathrm{~min}$. Cells were then washed three times with PBS and dyed with DAPI for $5 \mathrm{~min}$, prior to being washed a 
further three times and analyzed using a confocal microscope (CarlZeiss LSM 710, Carl Zeiss, Oberkochen, Germany). Excitation and emission wavelengths were $539 \mathrm{~nm}$ for $\mathrm{Cy} 3$ and $460 \mathrm{~nm}$ for DAPI, respectively.

Cell cycle analysis of cytotoxicity. To evaluate cell cytotoxicity of peptide NLS and peptide derivative SPB-NLS, the cell cycle of different transfection groups was analyzed. Transfection was performed in accordance with the aforementioned method. Cells were harvested and washed three times with PBS $48 \mathrm{~h}$ after transfection. Cells were then treated with $5 \mu \mathrm{g} / \mathrm{ml}$ RNaseA for $10 \mathrm{~min}$ at room temperature, and stained with $5 \mu \mathrm{g} / \mathrm{ml}$ propidium iodide, a DNA-binding dye, for $2 \mathrm{~h}$ at room temperature. Flow cytometry was then used to analyze the cell cycle.

Validation of the function of SPB-NLS in goat mammary epithelial cells (GMECs).

Induction of GMECs and cell transfection. GMECs were seeded in six-well dishes (Costar), and grown to 50-60\% confluence. Prior to experiment, cells were cultured in induction media for one week to promote the synthesis of milk protein and fat. The induction media contained $1 \%$ Insulin-Transferrin-Selenium Supplement (Invitrogen Life Technologies), $5 \mathrm{mg} / \mathrm{ml}$ progesterone (ProSpec, East Brunswick, NJ, USA), 10-7 mol/l hydrocortisone (R\&D Systems, Minneapolis, MN, USA), $10 \mathrm{ng} / \mathrm{ml}$ ovine epithelial growth factor (ProSpec) and $5 \mathrm{mg} / \mathrm{ml}$ bovine estradiol (Sigma-Aldrich).

Transfection was performed using plasmid pIN, mediated by SPB-NLS. Cells were divided into three groups: a control group (non-transfection), a transfection group (transfected with plasmid pIN) and an SPB-NLS group (transfected with pIN mediated by SPB-NLS). The culture medium was changed to DMEM/F-12 containing $10 \%$ FBS $6 \mathrm{~h}$ after transfection. Cells were collected after $48 \mathrm{~h}$ for subsequent analysis.

Western blot analysis of IGF-1 protein expression. GMECs were lysed with RIPA buffer containing phosphatase and protease inhibitors (1 mM PMSF) on ice for $30 \mathrm{~min}$. Cell lysates were centrifuged at $14,000 \mathrm{x}$ g for $10 \mathrm{~min}$ at $4^{\circ} \mathrm{C}$. The proteins in the supernatant were separated using $4-15 \%$ SDS-PAGE, and transferred onto nitrocellulose membranes. Membranes were blocked with TBS and Tween 20 buffer containing $5 \%$ goat serum at room temperature for $2 \mathrm{~h}$. Membranes were subsequently incubated with mouse anti-human IGF-1 monoclonal antibody at $4^{\circ} \mathrm{C}$ for $18 \mathrm{~h}$ and then rabbit anti-mouse IgG-HRP secondary antibody. Protein expression was detected using an enhanced chemiluminescence detection system (Amersham Pharmacia Biotech, Inc.), using $\beta$-actin as a loading control. All bands from the western blotting were analyzed using Quantity One software (Bio-Rad) to verify their relative expression.

Statistical analysis. The transfection efficiency and cell cytotoxicity were analyzed using one-way analysis of variance followed by a Least Significant Difference post hoc test. $\mathrm{P}<0.05$ was considered to indicate a statistically significant difference. All results are expressed as the mean \pm standard error of the mean.
A

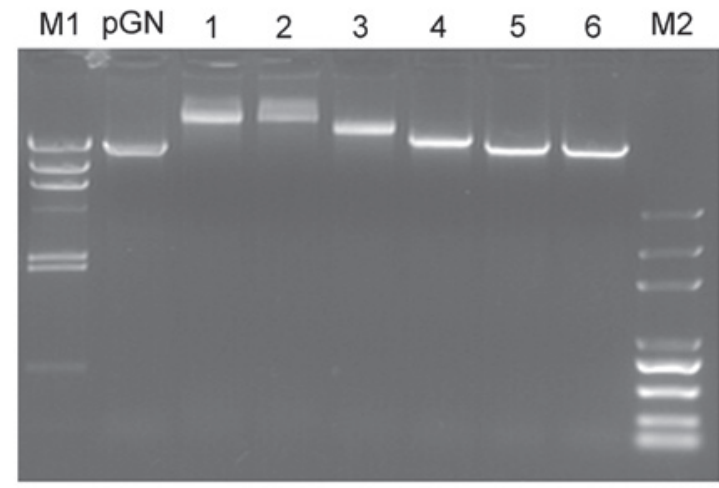

(NLS/pGN)

B

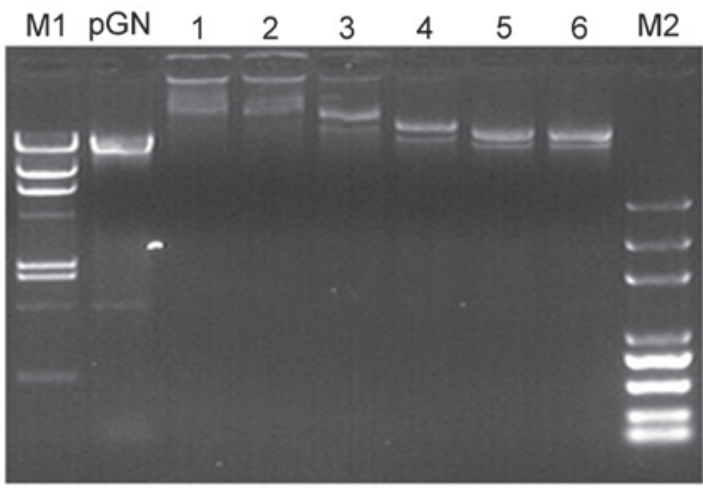

(SPB-NLS/pGN)

Figure 2. Gel retardation assay. Electrophoresis of different concentrations of (A) NLS and (B) SPB-NLS binding to plasmid pGN. (A) Lane 1, naked DNA; lanes 2-7, varying molar ratios of NLS/pGN $\left(5 \times 10^{4}, 2 \times 10^{4}, 1 \times 10^{4}, 5 \times 10^{3}, 1 \times 10^{3}\right.$ and $5 \times 10^{2}$ ); M1, Marker $\lambda$ DNA/HindIII; M2, Marker DNA/Trans2K Plus. (B) Lane 1, naked DNA; lanes 2-7, varying molar ratios of SPB-NLS/pGN $\left(5 \times 10^{4}, 2 \times 10^{4}, 1 \times 10^{4}, 5 \times 10^{3}, 1 \times 10^{3}\right.$ and $\left.5 \times 10^{2}\right)$; M1, Marker $\lambda$ DNA/HindIII M2, Marker DNA/Trans2K Plus. NLS, nuclear localization sequence; SBP-NLS, succinimidyl-[4-(psoralen-8-yloxy)]-butyrate-NLS.

\section{Results}

Gel retardation assay. A prerequisite for transfection-enhancing agents is their ability to bind nucleic acids. In order to determine the optimal conditions for complex formation between plasmid DNA ( $\mathrm{pGN}, 1 \mu \mathrm{g}$ ) and NLS peptide or peptide derivative SPB-NLS, different molar ratios (between $5 \times 10^{2}$ and $5 \times 10^{4}$ ) of NLS and SPB-NLS were used. The migration of the complexes was detected using a gel retardation assay (Fig. 2). Compared with naked plasmid DNA (pGN, lane 1), the NLS/DNA and SPB-NLS/DNA showed varying degrees of retardation (lanes 2-7). The results from the gel electrophoresis demonstrated that the complexes (NLS/DNA and SPB-NLS/DNA) were strongly retarded at molar ratios of $5 \times 10^{4}$ and $2 \times 10^{4}$, respectively, whilst the complexes at molar ratios of $5 \times 10^{2}$ and $1 \times 10^{3}$ exhibited no or little retardation. At a molar ratio $5 \times 10^{3}$ the complexes showed optimal retarded mobility. Additionally, NLS and SPB-NLS at a molar ratio of $5 \times 10^{3}$ did not affect the mobility of plasmid DNA. Therefore, a molar ratio of $5 \times 10^{3}$ was used in subsequent experiments.

Analysis of the transfection efficiency of NLS and SPB-NLS qPCR analysis. Transfection efficiency of NLS and SPB-NLS was analyzed with plasmid pGN. The results from the qPCR 
Table II. Relative GH mRNA expression levels in different groups.

Treatment

\begin{tabular}{|c|c|c|c|c|c|}
\hline Parameter & Blank & $\begin{array}{c}\text { Control } \\
\text { (liposome) }\end{array}$ & $\begin{array}{l}\text { Liposome }+ \\
\text { pGN }\end{array}$ & $\begin{array}{l}\text { Liposome + } \\
\text { NLS + pGN }\end{array}$ & $\begin{array}{c}\text { Liposome + } \\
\text { SPB-NLS + pGN }\end{array}$ \\
\hline $\begin{array}{l}\text { Relative GH } \\
\text { mRNA expression }\end{array}$ & $1.039 \pm 0.349$ & $2.182 \pm 0.329$ & $11.006 \pm 1.909$ & $18.644 \pm 1.534^{\mathrm{a}}$ & $47.648 \pm 4.620^{\mathrm{b}}$ \\
\hline
\end{tabular}

GH, growth hormone; NLS, nuclear localization sequence; SBP-NLS, succinimidyl-[4-(psoralen-8-yloxy)]-butyrate-NLS. ${ }^{\text {P }}<0.05$, vs. liposome + pGN; ${ }^{\mathrm{b}} \mathrm{P}<0.01$, vs. liposome + pGN.
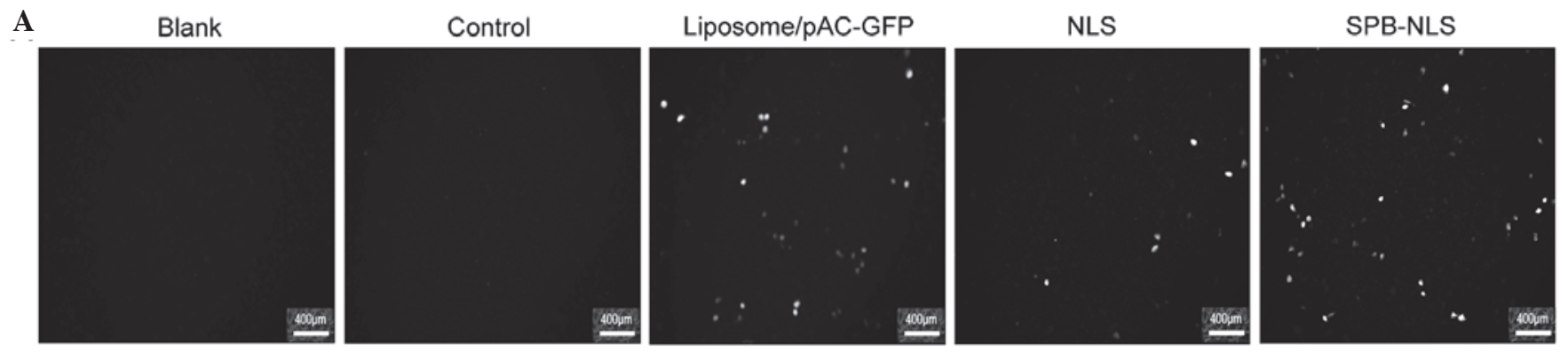

B
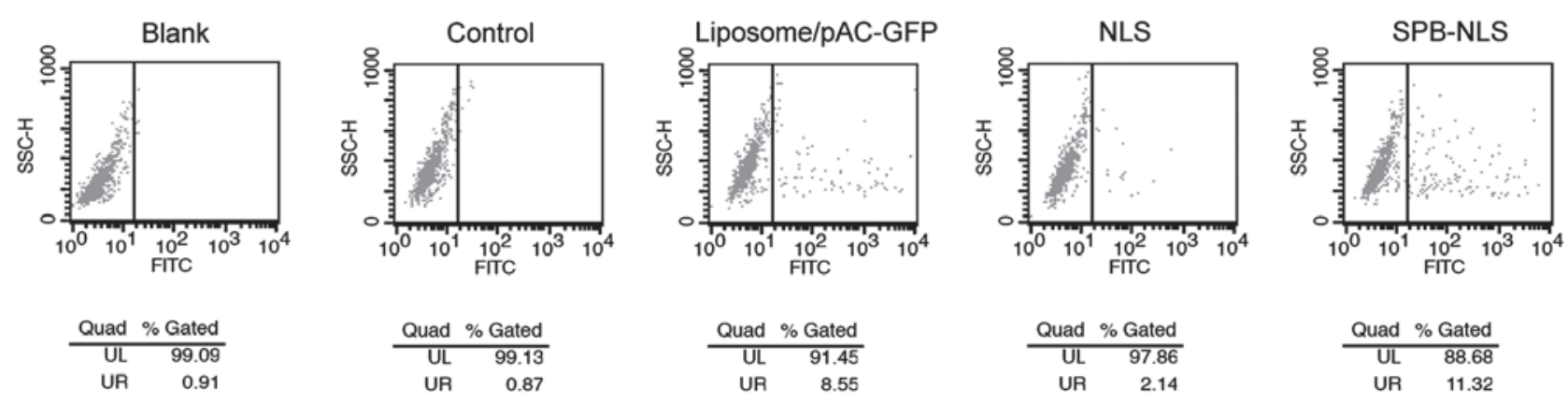

C
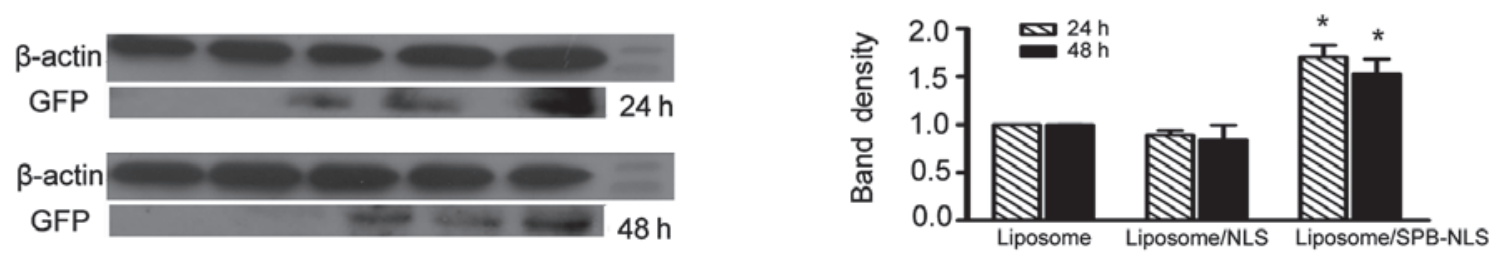

Figure 3. Analysis of transfection efficiency. (A) Fluorescence microscopy results of transfection efficiency in blank, control, liposome, NLS and SPB-NLS groups. (B) Flow cytometry results of transfection efficiency in the blank, liposome, NLS and SPB-NLS groups. (C) Western blot analysis of GFP expression levels. Lane 1, blank group; lane 2, negative control (cells transfected with plasmid pGN); lane 3, liposome group (cells transfected with plasmid pAC-GFP-N1 by liposome); lane 4, liposome/NLS group (cells transfected with plasmid pAC-GFP-N1 by liposome and NLS); lane 5, liposome/SPB-NLS group (cells transfected with plasmid pAC-GFP-N1 by liposome and SPB-NLS). The data are presented as the mean \pm the standard error of the mean of triplicate wells from three independent experiments. "P<0.05 vs. liposome group. GFP, green fluorescent protein; NLS, nuclear localization sequence; SBP-NLS, succinimidy 1-[4-(psoralen-8-yloxy)]-butyrate-NLS; FITC, fluorescein isothiocyanate; SSC-H, side scatter pulse height; UL, upper left; UR, upper right.

showed that the expression levels of GH mRNA were significantly higher in the liposome/pGN group $(11.006 \pm 1.909)$ than those in the blank $(1.039 \pm 0.349)$ and control $(2.182 \pm 0.329)$ groups. GH mRNA expression was also significantly higher in the NLS-mediated group (18.644 \pm 1.534$)$ and

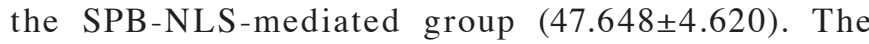
SPB-NLS-mediated group exhibited peak levels of GH mRNA. The expression levels of GH mRNA in the NLS and SPB-NLS groups were $69 \%$ and $330 \%$ greater than those in the liposome/pGN group, respectively (Table II).
Fluorescence microscopy and flow cytometry. Achieving high transgenic expression efficiency was the ultimate aim of the present study. An in vitro gene transfer assay was performed with plasmid pAC-GFP-N1, and fluorescence microscopy was used to evaluate the transfection efficiency of NLS and SPB-NLS. Untreated cells served as the blank group and cells transfected with plasmid pGN served as the negative control group. The results from the fluorescence microscopy showed that there was no GFP expression in the blank and negative groups. Compared with the blank group, only small 
A

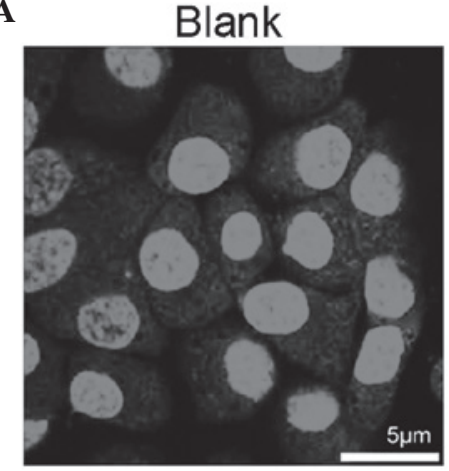

D

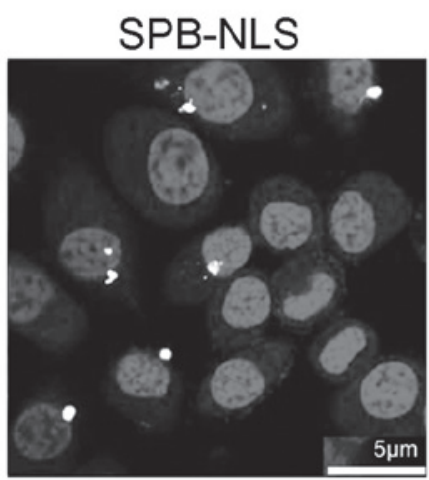

B

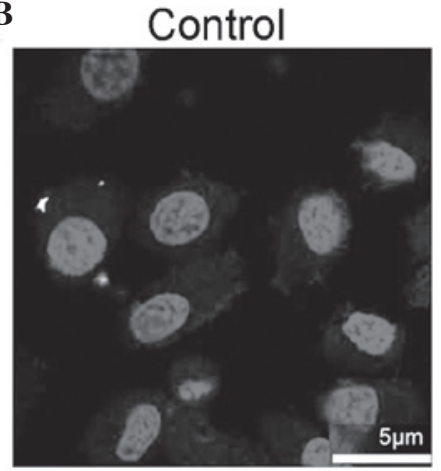

C

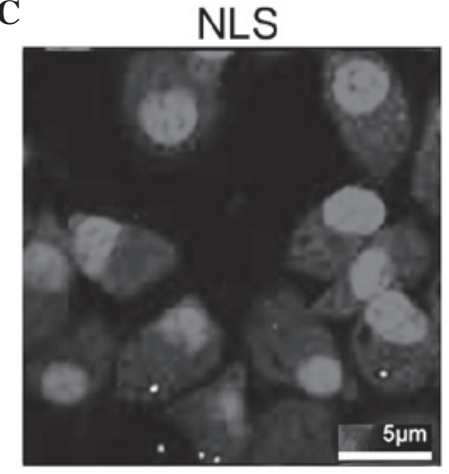

$\mathbf{E}$

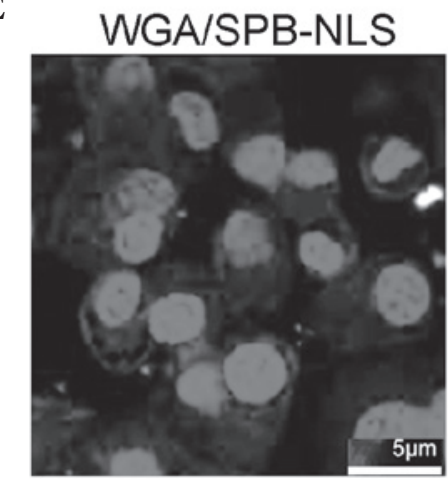

Figure 4. Confocal microscopy detection of plasmid DNA (white). Plasmid was detected using a Cy 3 labeling kit and nuclei were stained using DAPI (grey). Stained cells were examined using confocal microscopy (magnification, x630). (A) Blank group; (B) control group (liposome/Cy3-pGN group); (C) liposome/NLS/Cy3-pGN group; (D) liposome/SPB-NLS/Cy3-pGN group; (E) WGA/liposome/SPB-NLS/Cy3-pGN group (WGA-blocked group). NLS, nuclear localization sequence; SBP-NLS, succinimidyl-[4-(psoralen-8-yloxy)]-butyrate-NLS; WGA, wheat-germ agglutinin.

areas of fluorescence were observed in the NLS group, similar to those in the group treated with liposome/pAC-GFP-N1. However, large areas of fluorescence were observed in the SPB-NLS group, indicating that GFP was highly expressed in the SPB-NLS group (Fig. 3A).

Further analysis using flow cytometry demonstrated that the percentage of cells expressing GFP in the blank, negative (liposome) and control (liposome/pGN) groups was $0.91,1.32$ and $0.87 \%$, respectively. The percentage of cells expressing GFP in the positive group (liposome/pAC-GFP-N1) was $8.55 \%$. The percentage of positive cells was greater in the SPB-NLS group $(11.32 \%)$ than that in the positive group. However, the percentage of positive cells in the NLS group (2.14\%) was lower than that in the positive group (Fig. 3B) Data analysis demonstrated that the number of cells positively expressing GFP in the SPB-NLS group was increased by $32.4 \%$, but decreased by $75 \%$ in the NLS group, compared with the positive group. These results are consistent with the results from the fluorescence microscopy analysis.

Western blot analysis. The results from the fluorescence microscopy and flow cytometry demonstrated that SPB-NLS has an important role in promoting gene transfer. To confirm these results, the expression of GFP in the SPB-NLS and NLS groups was investigated. The results of the western blot analysis demonstrated a significant increase in GFP expression in the SPB-NLS group (relative band density, 1.70 \pm 0.13 , $24 \mathrm{~h} ; 1.53 \pm 0.16,48 \mathrm{~h}$ ) compared with that in the liposome group (relative band density, $1.00 \pm 0.001,24 \mathrm{~h} ; 1.00 \pm 0.001$, $48 \mathrm{~h})(\mathrm{P}<0.05$; Fig. 3C). By contrast, the expression of GFP

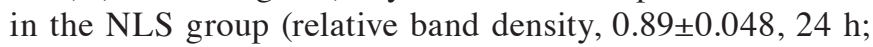

$0.84 \pm 0.16,48 \mathrm{~h})$ was slightly lower than that in the liposome group (Fig. 3C).

Confocal microscopy to identify the delivery of plasmid DNA. Since nuclear import efficiency greatly influences transgenic expression efficiency, it was investigated whether NLS and SPB-NLS facilitated nuclear entry of plasmid DNA. Confocal microscopy analysis demonstrated that no plasmid DNA (red) was present in the nucleus in the control group (Fig. 4A). Merged images with Cy3 and DAPI staining revealed that a small number of red plasmids were present in the perinuclear area in the liposome/pGN group (Fig. 4B). In the NLS group, there was an increase in the number of plasmids in the cell, indicating that NLS enhanced plasmid transport into the cytoplasm. However, in the NLS group few or no plasmids entered the nucleus (Fig. 4C). By contrast, in the SPB-NLS group there was an increase in the number of plasmids in the nucleus, demonstrating that SPB-NLS enhanced entry of plasmid DNA into the nucleus (Fig. 4D). To confirm whether SPB-NLS was interacting with the nuclear pore complex (NPC), WGA was used to specifically block the nuclear pores. When cells were treated with WGA, few plasmids were observed in the cytoplasm and none were observed in the nucleus. Therefore, peptide derivative SPB-NLS increases transfection efficiency by interacting with the NPC (Fig. 4E).

Cells cytotoxicity analysis. An optimized gene delivery system should be able to improve transfection efficiency without any toxic effects. The cell cycle is a good indicator of cell viability. Flow cytometric analysis revealed marked differences in the 
Table III. Cell cycle analysis of cytotoxicity.

\begin{tabular}{lcccc}
\hline Treatment & G1 $(\%)$ & S $(\%)$ & G2 $(\%)$ & Apoptosis $(\%)$ \\
\hline Blank & $61.41 \pm 0.42$ & $31.58 \pm 0.89$ & $7.01 \pm 0.47$ & $1.11 \pm 0.29$ \\
Liposome/pGN & $56.79 \pm 0.87$ & $38.93 \pm 2.30$ & $4.29 \pm 1.43$ & $2.21 \pm 0.59$ \\
Liposome/SPB-NLS/pGN & $57.19 \pm 0.13$ & $38.70 \pm 1.04$ & $4.12 \pm 0.91$ & $1.74 \pm 0.29$
\end{tabular}

SBP-NLS, succinimidyl-[4-(psoralen-8-yloxy)]-butyrate-nuclear localization sequence.

A

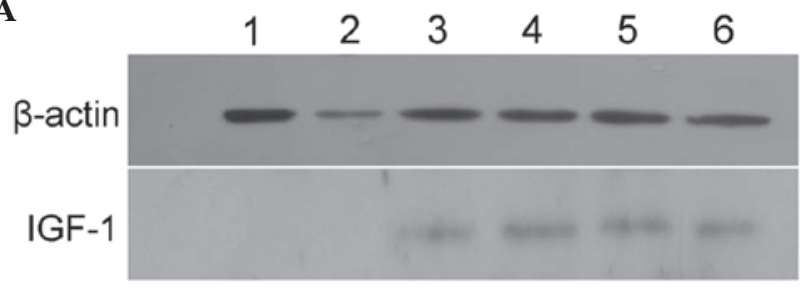

B

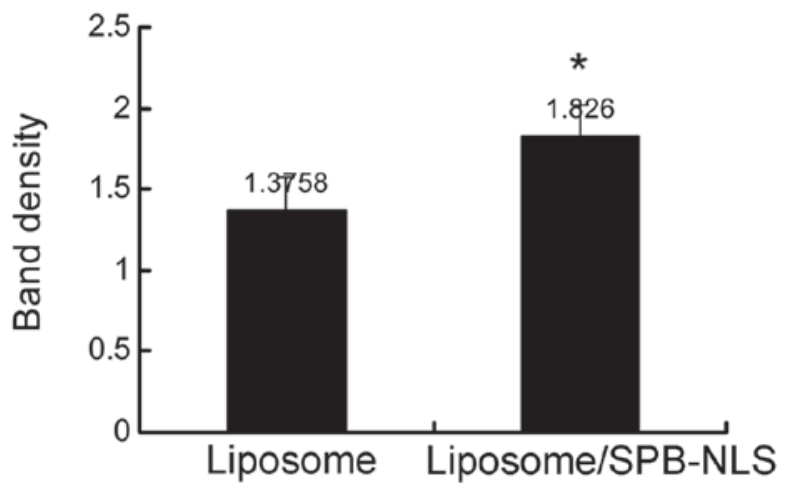

Figure 5. Western blot analysis of IGF-1 expression in GMECs. IGF-1 expression levels were assessed in GMECs using western blot analysis. Plasmid pIN was transfected into GMECs to evaluate the transfection efficiency of SPB-NLS. (A) Results from the western blot anaylis. Lanes 1 and 2, blank group; lanes 3 and 4, liposome group (cells transfected with plasmid pIN by liposome); lanes 5 and 6, SPB-NLS group (cells transfected with plasmid pIN by liposome and SPB-NLS. (B) Relative band density of the liposome and the SPB-NLS groups from the results of the western blot analysis. The results are presented as the mean \pm the standard error of the mean of double wells from three independent experiments. ${ }^{*} \mathrm{P}<0.05$ vs. liposome group. IGF-1, insulin-like growth factor 1; GMECs, goat mammary epithelial cells; SBP-NLS, succinimidyl-[4-(psoralen-8-yloxy)]-butyrate-nuclear localization sequence.

cell cycle between the blank and liposome/pGN groups in the G1 and S phases (Table III). Data analysis demonstrated that liposome affected the G1, S and G2 phases, indicating that liposome treatment may have a slight cytotoxic effect on cells. The apoptosis percentages of the SPB NLS and NLS groups revealed similarities with the liposome group, indicating that SPB NLS and NLS may also have a slight toxic effect.

Analysis of SPB-NLS function in GMECs. Previous experiments demonstrated that SPB-NLS improved the transfection efficiency in the Bcap-37 cell line. To investigate whether SPB-NLS may be used to alleviate problems in the development of transgenic animals, plasmid pIN was transfected into GMECs to evaluate the transfection efficiency. Transfection of pIN into GMECs was confirmed using immunohistochemistry and PCR (data not shown). IGF-1 protein was found to be expressed in all plasmid pIN-transfected groups. In addition, the SPB-NLS group (relative band density, 1.826 \pm 0.191 ) exhibited greater IGF-1 protein expression than the liposome group (relative band density, 1.376 \pm 0.204 ) (Fig. 5). These results further indicated that SPB-NLS enhances transfection of large DNA molecules (plasmid pIN).

\section{Discussion}

The present study aimed to investigate methods to improve gene transfer efficiency and to characterize the mechanism of plasmid import into transfected cells in order to improve DNA delivery in vivo. The study also aimed to develop a convenient delivery system for functional gene transfer and efficient screening for the development of transgenic animals. All macromolecules enter or exit the nucleus through the NPC (15). Similarly, plasmid DNA is transported into the nuclei of non-dividing cells via the NPC. DNA fragments $>1 \mathrm{~kb}$ are unable to enter the nuclei, whilst those $<1 \mathrm{~kb}$ readily pass through the NPC $(16,17)$. In contrast to what has previously been demonstrated in intact cells, plasmids $\leq 15 \mathrm{~kb}$ are transported into the nuclei upon cell division (18). It is well established that the NLS has an important role in DNA transport into the nucleus. In this study it was investigated whether large DNA molecules (such as plasmids pIN, $23 \mathrm{~kb}$, and pGN, $23 \mathrm{~kb}$ ) were able to pass in and out of the nucleus with the aid of NLS/SPB-NLS to allow continued expression.

The interaction between the transfection complex, NLS/SPB-NLS, and DNA was first evaluated. Two peptides were designed to condense DNA and enhance delivery into the nucleus. Previous studies have found that NLS peptides do not markedly enhance plasmid DNA nuclear entry efficiency and that chemical modifications of DNA even reduce the transcription efficiency or stability in the nucleus (10). Consistent with this, the results from the present study demonstrated that NLS peptide downregulated gene expression efficiency: Fluorescence microscopy and western blot analysis showed that the NLS group expressed less GFP than the liposome/pGN group (10). The low expression efficiency in the NLS group may have been due to a lack of binding. Enhancing the binding of DNA with NLS was therefore the first challenge. A previous study by Lechardeur and Lukacs (2) found that single- and double-stranded plasmids were degraded with an apparent half-life of between 50 and 90 min. Several different methods have been used to attach NLS to plasmid DNA, including ionic interaction (19), covalent attachment (7) and peptide nucleic 
acid clamp (20), and have been shown to significantly improve nuclear transport efficiency. However, increased transport into the nucleus does not necessarily correlate with increased levels of protein expression (21). Ciolina et al (22) demonstrated that microinjected DNA with covalently attached NLS raised transfection efficiency by $\leq 160 \%$ compared with unmodified DNA (22); however, expression decreased by $60 \%$ with plasmid bearing 43 NLS peptides. The results from the present study showed that the expression levels of GH mRNA in the NLS group were increased by $69 \%$ compared with those in the liposome/pGN group, but the percentage of positive cells (2.14\%) and the GFP protein expression levels (relative band density, $0.89 \pm 0.048,24 \mathrm{~h} ; 0.84 \pm 0.16,48 \mathrm{~h}$ ) were lower than those in the liposome/pGN group. In combination these results suggest that covalent binding of NLS to DNA leads to transcriptional inactivation, possibly due to over-tight binding (10). Therefore, non-covalent modification of DNA may be an alternative (23). It has been suggested that SPB has the potential to non-covalently modify target molecules without affecting gene expression (24). In the present study it was demonstrated using flow cytometry and western blot analysis that GFP expression was elevated in the SPB-NLS group, indicating that SPB-NLS has high biological activity.

qPCR was performed to quantitatively evaluate the transfection efficiency of NLS and SPB-NLS. The results revealed that SPB-NLS notably improved gene transfer efficiency. The mRNA expression levels of GH in the NLS and SPB-NLS groups were higher than those in the liposome/pGN group, which is consistent with the results by Jain and Gewirtz (25). The results from the present study demonstrate that the NLS group had higher mRNA expression levels of GH than the liposome/pGN group, indicating that NLS has an important role in gene transfer. The exact mechanism of SPB-NLS-mediated gene delivery has yet to be elucidated; however, the results of the present study show that the addition of NLS and SPB-NLS may affect gene transcription efficiency. Further investigation is required, however, to understand the mechanism by which SPB-NLS affects transfection.

In order to assess the effect of NLS and SPB-NLS on gene expression, GFP expression levels were analyzed using fluorescence microscopy, flow cytometry and western blotting. Flow cytometry quantitatively estimates gene expression and accurately determines fluorescent intensity. Similar results were found using the different methods. It was found that GFP expression was significantly increased in the SPB-NLS group but decreased in the NLS group compared with that in the liposome/pGN group. Similarly, high GH mRNA transcription efficiency along with low GFP expression efficiency was observed in the NLS group. This phenomenon may have been a result of the content of the DNA transferred into the nucleus and due to the plasmid DNA being prevented from being degraded by nucleases.

Lipid-mediated transfection requires endocytosis. Unprotected DNA in the cytoplasm is degraded by resident cytosolic nucleases (26). Much of the transferred DNA is retained in endosomes, escapes to the cytoplasm and enters into the nucleus at low rates, which limits the efficiency of liposome-mediated gene transfer (27). The results of the present study demonstrated that in the liposome/pGN group plasmids were located around the nuclear membrane; however, in the SPB-NLS group, plasmid DNA was observed in the nucleus. Since the nuclear envelope is an important barrier in transfection, the mechanism underlying the interaction of SPB-NLS with the nuclear envelope was investigated. The position of labeled plasmid DNA was analyzed using confocal microscopy (28). The results revealed that the labeled plasmids were delivered to the nucleus in the SPB-NLS group. In the liposome/pGN group, however, the plasmids remained around the nuclear envelope. To determine whether SPB-NLS was interacting with the NPC, WGA was used. WGA cross-links with phenylalanine-rich repeat motifs in the NPC, specifically inhibiting the exchange of material between the nucleus and the cytoplasm. Following WGA treatment, no labeled plasmid was observed in the nucleus, further demonstrating that SPB-NLS affects the formation of the NPC by interacting with nuclear envelope. Contradictory to our study, a previous report suggested that a random-walk diffusion of DNA molecules was likely to be inefficient and slow (29). However, naked DNA does not remain free in the nucleus as histones rapidly assemble transferred DNA into chromatin-like structures, thus providing a mechanism for pulling and condensing the filamentous molecule into the nucleus.

Unless plasmid DNA enters the nucleus, no gene integration or replication of any plasmid DNA occurs. NLS mediates the trafficking from the cytoplasm into the nucleus. In the present study it was found that SPB-NLS significantly enhanced gene transfer and expression efficiency. Although high transfection efficiency was demonstrated, the mechanism by which SPB-NLS enhances transfection has yet to be fully elucidated. In conclusion, in this study it was shown that SPB-NLS, as a transfection-enhancing agent, may improve the transport of large molecular DNA into the nucleus and provides a 'fixed target' in nuclear trafficking. In the future this may provide a safe and alternative strategy for rapid and efficient screening of transgenic positive clones.

\section{Acknowledgements}

This study was supported by grants from the National Animal Transgenic Breeding Grand Project (nos. 2011ZX08008-004 and 2013ZX08008-004) and the Priority Academic Program Development of Jiangsu Higher Education Institutions (PAPD).

\section{References}

1. Whitelaw CB, Farini E and Webster J: The changing role of cell culture in the generation of transgenic livestock. Cytotechnology 31: 3-8, 1999.

2. Lechardeur D and Lukacs GL: Intracellular barriers to non-viral gene transfer. Curr Gene Ther 2: 183-194, 2002.

3. Leong KW, Mao HQ, Truong-Le VL, Roy K, Walsh SM and August JT: DNA-polycation nanospheres as non-viral gene delivery vehicles. J Control Release 53: 183-193, 1998.

4. Ludtke JJ, Sebestyén MG and Wolff JA: The effect of cell division on the cellular dynamics of microinjected DNA and dextran. Mol Ther 1: 579-588, 2002.

5. Munkonge FM, Dean DA, Hillery E, Griesenbach U and Alton EW: Emerging significance of plasmid DNA nuclear import in gene therapy. Adv Drug Deliv Rev 55: 749-760, 2003.

6. Kamiya $\mathrm{H}$, Tsuchiya $\mathrm{H}$, Yamazaki $\mathrm{J}$ and Harashima $\mathrm{H}$ : Intracellular trafficking and transgene expression of viral and non-viral gene vectors. Adv Drug Deliv Rev 52: 153-164, 2001. 
7. Zanta MA, Belguise-Valladier P and Behr JP: Gene delivery: a single nuclear localization signal peptide is sufficient to carry DNA to the cell nucleus. Proc Natl Acad Sci USA 96: 91-96, 1999.

8. Subramanian A, Ranganathan P and Diamond SL: Nuclear targeting peptide scaffolds for lipofection of nondividing mammalian cells. Nat Biotechnol 17: 873-877, 1999.

9. Brandén LJ, Mohamed AJ and Smith CI: A peptide nucleic acid-nuclear localization signal fusion that mediates nuclear transport of DNA. Nat Biotechnol 17: 784-787, 1999.

10. Tanimoto M, Kamiya H, Minakawa N, Matsuda A and Harashima H: No enhancement of nuclear entry by direct conjugation of a nuclear localization signal peptide to linearized DNA. Bioconjug Chem 14: 1197-1202, 2003.

11. Man N, Yu L, Zheng F, Li Y and Wen LP: Efficient gene transfer to rat fetal osteoblastic cells by synthetic peptide vector system. Protein Pept Lett 16: 368-372, 2009.

12. Smith J, Guidry J and Wittung-Stafshede P: Novel 'three-in-one' peptide device for genetic drug delivery. Protein Pept Lett 10: 1-7,2003.

13. Lin J, Yu Q, Zhang Q and Yang Q: Construction of mammary gland specific expression plasmid pIN and its expression in vitro and in vivo. Afr J Biotechnol 11: 7038-7045, 2012.

14. $\mathrm{Hu} \mathrm{H}$, Wang J, Bu D, et al: In vitro culture and characterization of a mammary epithelial cell line from Chinese Holstein dairy cow. PLoS One 4: e7636, 2009.

15. Gasiorowski JZ and Dean DA: Mechanisms of nuclear transport and interventions. Adv Drug Deliv Rev 55: 703-716, 2003.

16. Ludtke JJ, Zhang G, Sebestyén MG and Wolff JA: A nuclear localization signal can enhance both the nuclear transport and expression of $1 \mathrm{~kb}$ DNA. J Cell Sci 112: 2033-2041, 1999.

17. Sebestyén F, Szendrei G, Mák M, et al: Coloured peptides: synthesis, properties and use in preparation of peptide sub-library kits. J Pept Sci 4: 294-299, 1998.

18. Wildeman AG: Regulation of SV40 early gene expression. Biochem Cell Biol 66: 567-577, 1988.
19. Akita H, Tanimoto M, Masuda T, et al: Evaluation of the nuclear delivery and intra-nuclear transcription of plasmid DNA condensed with micro (mu) and NLS-micro by cytoplasmic and nuclear microinjection: a comparative study with poly-L-lysine. J Gene Med 8: 198-206, 2006.

20. Díaz-Mochón JJ, Bialy L, Watson J, Sánchez-Martín RM and Bradley M: Synthesis and cellular uptake of cell delivering PNA-peptide conjugates. Chem Commun (Camb) 3316-3318, 2005.

21. Leahy P, Carmichael GG and Rossomando EF: Novel biotinylated plasmid expression vectors retain biological function and can bind streptavidin. Bioconjug Chem 7: 545-551, 1996.

22. Ciolina C, Byk G, Blanche F, Thuillier V, Scherman D and Wils P: Coupling of nuclear localization signals to plasmid DNA and specific interaction of the conjugates with importin alpha. Bioconjug Chem 10: 49-55, 1999.

23. Boulanger C, Di Giorgio C and Vierling P: Synthesis of acridine-nuclear localization signal (NLS) conjugates and evaluation of their impact on lipoplex and polyplex-based transfection. Eur J Med Chem 40: 1295-1306, 2005.

24. Grosse S, Thévenot G, Monsigny M and Fajac I: Which mechanism for nuclear import of plasmid DNA complexed with polyethylenimine derivatives? J Gene Med 8: 845-851, 2006.

25. Jain PT and Gewirtz DA: Enhancement of liposomal gene delivery in human breast cancer cells by dimethyl sulfoxide. Int $\mathrm{J}$ Mol Med 1: 609-611, 1998

26. Braun K, von Brasch L, Pipkorn R, et al: BioShuttle-mediated plasmid transfer. Int J Med Sci 4: 267-277, 2007.

27. Mehier-Humbert S, Bettinger T, Yan F and Guy RH: Ultrasound-mediated gene delivery: kinetics of plasmid internalization and gene expression. J Control Release 104: 203-211, 2005.

28. Zhou M, Liu H, Xu X, et al: Identification of nuclear localization signal that governs nuclear import of BRD7 and its essential roles in inhibiting cell cycle progression. J Cell Biochem 98: 920-930, 2006.

29. Cereghini S and Yaniv M: Assembly of transfected DNA into chromatin: structural changes in the origin-promoter-enhancer region upon replication. EMBO J 3: 1243-1253, 1984. 